\title{
Use of indomethacin for pain relief following scleral buckling surgery
}

\author{
S A Sadiq, L Stevenson, C Gorman, G M Orr
}

\begin{abstract}
Backgroundlaims-Patients undergoing scleral buckling and cryotherapy suffer from mild to moderate postoperative pain. As good pain relief facilitates postoperative ocular examination, as well as patient comfort and recovery, the authors designed a prospective randomised double masked trial to evaluate the efficacy of indomethacin as a satisfactory analgesic for such patients.

Method-Patients with a primary uncomplicated rhegmatogenous retinal detachment requiring scleral buckling and cryotherapy were randomly allocated to receive either indomethacin or placebo. A rectal suppository was administered 2 hours before surgery, followed by two capsules twice daily for 10 days. Pain relief was assessed with a linear graphic rating scale at the end of each day. Supplementary analgesia was allowed and recorded. Results-12 patients received indomethacin (group A) and 16 received placebo (group B). The extent of surgery was similar in both groups. One patient in group $A$, and two in group $B$ withdrew after 3 days. The pain scores were converted to changes from the baseline (score on day 1), and the area under the curve calculated for each patient. The means of the areas were analysed with the MannWhitney test and showed that indomethacin caused a statistically significant reduction in pain score, both at 3 days $(p=0.04)$ and at 10 days $(p=0.014)$. There was no statistically significant difference in extra analgesic requirements between the two groups $(p=0.2)$.
\end{abstract}

Conclusions-Indomethacin is recommended for short to medium term pain relief following scleral buckling and cryotherapy.

(Br F Ophthalmol 1998;82:429-431)

Retinal detachment is a common presenting cause of loss of vision and requires surgical intervention shortly after presentation. Uncomplicated surgery usually involves a scleral buckling procedure with cryotherapy to the retinal defect, and this is still the commonest type of surgery performed for retinal detachments. Drainage of subretinal fluid and injection of air may also be required.

Patients undergoing scleral buckling surgery do suffer from mild to moderate postoperative pain due to the inflammation caused by the procedure. Scleral buckling and cryotherapy induce a breakdown of blood-ocular barriers and release of prostaglandins, with resultant inflammation and pain. Personal experience of many vitreoretinal surgeons shows that nonsteroidal anti-inflammatory drugs (NSAIDs, given as a rectal suppository preoperatively and as oral doses postoperatively) seem to provide adequate analgesia for these patients, although this has not been confirmed by a controlled study. We therefore designed a prospective randomised double masked trial to evaluate the efficacy of indomethacin as a satisfactory analgesic for patients undergoing scleral buckling and cryotherapy.

\section{Methods}

Ethics committee approval was obtained before the start of the study. Informed consent was obtained from patients suffering from an uncomplicated rhegmatogenous retinal detachment requiring a scleral buckle and cryotherapy. Patients with a history of previous ocular surgery were excluded. Patients were also excluded if they had a history of any of the following: known allergy to NSAIDs and aspirin, peptic ulcer/gastrointestinal bleeding, severe cardiac/pulmonary/liver/kidney disease, malignant hypertension, haemorrhagic disorders, known or suspected pregnancy/nursing mothers, use of an NSAID before surgery, psoriasis, asthma, concomitant treatment with lithium, epilepsy, parkinsonism, or proctitis/ haemorrhoids. Patients were withdrawn from the study if adverse effects possibly attributed to indomethacin occurred.

Patients were randomly allocated to receive either indomethacin or a placebo. Randomisation was performed by the pharmacy department, who then supplied indomethacin $25 \mathrm{mg}$ capsules, indomethacin $100 \mathrm{mg}$ rectal suppositories, placebo capsules, and placebo rectal suppositories. The indomethacin and placebo preparations had the same appearance, and coded numbers were used. The code was not broken until the study was completed. Care was taken to ensure that each suppository was inserted correctly.

A rectal suppository was administered by nursing staff 2 hours before scleral buckling surgery while the patient was "nil by mouth". Following surgery, two capsules were prescribed twice daily for 10 days from the first postoperative day. No premedication was given before anaesthesia. During anaesthesia no long acting analgesics were administered. Short acting analgesics-for example, fentanyl, were allowed.

Pain relief was assessed using a $10 \mathrm{~cm}$ linear graphic rating scale. This was a visual analogue scale with descriptive terms placed at intervals
Accepted for publication 5 November 1997 
Table 1 Details of patients

\begin{tabular}{lcc}
\hline & $\begin{array}{l}\text { Indomethacin } \\
\text { (Group A) }\end{array}$ & $\begin{array}{l}\text { Placebo } \\
\text { (Group B) }\end{array}$ \\
\hline Males & 8 & 13 \\
Females & 4 & 3 \\
Right eye operated & 6 & 8 \\
Left eye operated & 6 & 8 \\
Mean age (years) & 61.8 & 53.4 \\
\hline
\end{tabular}

along the line, the validity of which has been previously demonstrated. ${ }^{1}$ The patients were asked to decide where on the scale they perceived pain or discomfort at the end of each day. This was recorded on the first postoperative day, and then daily for 10 days. The use of supplementary analgesia was allowed if required (but not NSAIDs), and the patients were asked to note when any additional analgesia was taken, as well as its name, dosage, and efficacy. Any adverse effects were also noted. The forms were collected at the first postoperative visit (usually at 14 days).

STATISTICAL METHODS

A two stage method involving summary measures was employed. ${ }^{2}$ In the first stage, the pain score at the end of day 1 was taken as the baseline, and the change from baseline was drawn as a curve for each patient. The area under the curve (AUC) was then calculated. In the second stage, the AUCs were treated as raw data, and analysed using the Mann-Whitney U test. The level of significance was chosen as $p<0.05$. The use of supplementary analgesia was analysed with the $\chi^{2}$ test with Yates's correction for small numbers.

\section{Results}

Twenty eight patients were entered into the study. Twelve patients received indomethacin (group A), and 16 received placebo (group B) (Table 1). The type and extent of surgery was the same in both groups, with a median of three muscles (range 2-4) being encircled during surgery. Only one patient in group A required peroperative prophylactic cryotherapy in the fellow eye. Two patients in group B received laser (none required cryotherapy).

In group $\mathrm{A}$, one patient withdrew because of nausea after day 3 . In group $B$, two patients withdrew after 3 days, one because of indigestion, while the second was admitted to the referring hospital for pain control where the medication (placebo) was replaced by Froben tablets with good analgesic effect. The results were thus analysed at 3 days for 28 patients, and at 10 days for 25 patients. The mean pain scores during the study are shown in Figure 1. In group $\mathrm{A}$, three out of 12 patients (25\%) required supplementary analgesia, and in group B, nine out of 16 patients (56\%), required supplementary analgesia.

Analysis of the AUCs showed that indomethacin caused a statistically significant reduction in pain scores, both at day 3 $(\mathrm{p}=0.04)$ and at day $10(\mathrm{p}=0.014)$. Analysis of supplementary medication showed that there was no statistically significant difference in extra analgesic requirements between those receiving indomethacin or placebo $(\mathrm{p}=0.2)$.

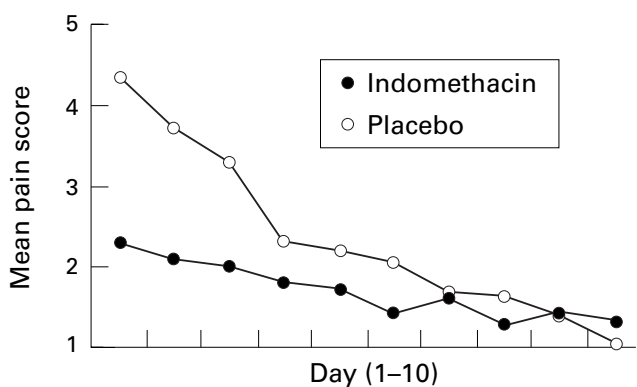

Figure 1 Mean pain score in patients receiving indomethacin or placebo.

\section{Discussion}

NSAIDs are one of the most commonly used agents in clinical practice today. In single doses, NSAIDs have analgesic activity comparable with that of paracetamol. ${ }^{3}$ In regular full dosage, NSAIDs have both a lasting analgesic and an anti-inflammatory effect, which makes them particularly useful for the treatment of continuous or regular pain associated with inflammation. Indomethacin has been shown to reduce postoperative pain scores following a wide variety of procedures such as lumbar disc prolapse, ${ }^{4}$ ureteral colic, ${ }^{5}$ and minor gynaecological or breast surgery. ${ }^{6}$ It can also reduce the need for opioid analgesics following thoracotomy ${ }^{7}$ and lumbar laminectomy. ${ }^{8}$ It is also efficacious as a spray for use in tendinitis, ${ }^{9}$ and has been used by ophthalmologists to treat posterior scleritis. ${ }^{10}$

Although indomethacin has been used empirically for patients undergoing retinal reattachment surgery (as per our protocol) for a while, we believe that this is the first controlled study investigating its role in pain relief. Conventional surgery involving scleral buckling and cryotherapy is still the commonest procedure used to repair a primary detachment in the majority of eye units. It is painful because it involves a certain amount of division of tissues in order to provide the required exposure, as well as manipulation and trauma to the globe and surrounding tissues during the process of explant siting and application. Cryotherapy itself can cause intense inflammation mediated by the release of prostaglandins and other mediators. In contrast, a vitrectomy approach retinal reattachment operation is considered to be a much less painful procedure with minimal trauma.

Although various studies have previously attempted to reduce postoperative pain following retinal surgery, they have mainly been concerned with short term pain relief immediately following surgery, ${ }^{11-13}$ unlike our study which investigates pain relief in the period following surgery and discharge from hospital (10 days). Although there is a reduction in pain, ${ }^{11}{ }^{12}$ the methods employed do involve further tissue manipulation, disruption, trauma, and swelling. We believe these ocular effects to be undesirable, hence our efforts to find a method of pain reduction which is easy, effective, and reduces medium term pain without further injury to the eye.

We have shown that indomethacin significantly reduces postoperative pain following 
conventional retinal reattachment surgery. The group of patients taking indomethacin also required fewer supplementary analgesics, although this was not statistically significant. Unfortunately all NSAIDs have side effects, mainly those affecting the gastrointestinal system. Differences in anti-inflammatory activity between different NSAIDs are small, with the main difference being in the incidence and type of side effects. ${ }^{3}$ Indomethacin is thought to have a higher rate of side effects than other NSAIDs ( $8 \%$ in our series), and we can see no reason why a "safer" NSAID may not be used with the same efficacy if there is worry regarding side effects. NSAIDs should of course be avoided in high risk patients, especially those specifically excluded by our protocol.

In conclusion, we recommend the use of indomethacin for short to medium term pain relief following scleral buckling and cryotherapy, but a careful medical history should be taken before its use. Good pain relief facilitates postoperative ocular examination, as well as patient comfort and recovery. We would suggest the prescription of a rectal suppository of indomethacin $100 \mathrm{mg}$ about 2 hours preoperatively, followed by indomethacin $50 \mathrm{mg}$ twice daily postoperatively for 10 days.
We thank Dr Kathryn Fielding, lecturer in medical statistics at Trent Institute of Health Services Research, for advice regarding the statistical methods.

1 Scott J, Huskisson E. Graphic representation of pain. Pain 1976;2:175-84.

2 Matthews J, Altman D, Campbell M, et al. Analysis of serial measurements in medical research. BMf 1990;300:230-5.

British National Formulary. Drugs used in the treatment of musculoskeletal and joint diseases. London: British Medical Association and the Royal Pharmaceutical Society of Great Britain, March 1997(33): 411.

4 Nissen I, Jensen K, Ohrstrom J. Indomethacin in the management of postoperative pain. Br f Anaesth 1992;69: 304-6.

5 Cordell W, Larson T, Lingeman J, et al. Indomethacin suppositories versus intravenously titrated morphine for the treatment of ureteral colic. Ann Emerg Med 1994;23:262-9.

6 Morley-Forster P, Newton P, Cook M. Ketorolac and post-operative pain. Can f Anaesth 1993;40:1126-30.

7 Pavy T, Medley C, Murphy D. Effect of indomethacin on pain relief after thoracotomy. Br F Anaesth 1990;65:624-7.

8 Rowe W, Goodwin A, Miller A. The efficacy of pre-operative controlled release indomethacin in the treatment of controlled release indomethacin in the treatment
post-operative pain. Curr Med Res Opin 1992;12:662-7.

post-operative pain. Curr Med Res Opin 1992;12:662-7.
9 Ginsberg F, Famaey J. Double-blind randomized crossover Ginsberg F, Famaey J. Double-blind randomized crossover
study of the percutaneous efficacy and tolerability of a topical indomethacin spray versus placebo in the treatment of tendinitis. F Int Med Res 1991;19:131-6.

10 Rosenbaum J, Robertson J. Recognition of posterior scleritis and its treatment with indomethacin. Retina 1993;13:17-21.

11 Gottfreothsdottir M, Gislason I, Stefansson E, et al. Effects of retrobulbar bupivacaine on post-operative pain and nausea in retinal detachment surgery. Acta Ophthalmol 1993;71:544-7.

12 Bourke R, Dowler J, Heyworth P, et al. Extraconal bupivacaine in scleral buckling procedures. Retina 1996;16: $240-5$.

13 Davis A, Wilkins M, Elliott A. Failure of topical bupivacaine to relieve pain after vitreoretinal surgery. Eye 1994;8:714-6. 